\title{
Basic Riemannian Geometry
}

\author{
F.E. Burstall \\ Department of Mathematical Sciences \\ University of Bath
}

\section{Introduction}

My mission was to describe the basics of Riemannian geometry in just three hours of lectures, starting from scratch. The lectures were to provide background for the analytic matters covered elsewhere during the conference and, in particular, to underpin the more detailed (and much more professional) lectures of Isaac Chavel. My strategy was to get to the point where I could state and prove a Real Live Theorem: the Bishop Volume Comparison Theorem and Gromov's improvement thereof and, by appalling abuse of OHP technology, I managed this task in the time alloted. In writing up my notes for this volume, I have tried to retain the breathless quality of the original lectures while correcting the mistakes and excising the out-right lies.

I have given very few references to the literature in these notes so a few remarks on sources is appropriate here. The first part of the notes deals with analysis on differentiable manifolds. The two canonical texts here are Spivak [5] and Warner [6] and I have leaned on Warner's book in particular. For Riemannian geometry, I have stolen shamelessly from the excellent books of Chavel [I] and Gallot-Hulin-Lafontaine [3]. In particular, the proof given here of Bishop's theorem is one of those provided in [3].

\section{What is a manifold?}

What ingredients do we need to do Differential Calculus? Consider first the notion of a continuous function: during the long process of abstraction and generalisation that leads from Real Analysis through Metric Spaces to Topology, we learn that continuity of a function requires no more structure on the domain and co-domain than the idea of an open set.

By contrast, the notion of differentiability requires much more: to talk about the difference quotients whose limits are partial derivatives, we seem to require that the (co-)domain have a linear (or, at least, affine) structure. 
However, a moment's thought reveals that differentiability is a completely local matter so that all that is really required is that the domain and codomain be locally linear, that is, each point has a neighbourhood which is homeomorphic to an open subset of some linear space. These ideas lead us to the notion of a manifold: a topological space which is locally Euclidean and on which there is a well-defined differential calculus.

We begin by setting out the basic theory of these spaces and how to do Analysis on them.

\section{$1.1 \quad$ Manifolds}

Let $M$ be a Hausdorff, second countable円, connected topological space.

$M$ is a $C^{r}$ manifold of dimension $n$ if there is an open cover $\left\{U_{\alpha}\right\}_{\alpha \in I}$ of $M$ and homeomorphisms $x_{\alpha}: U_{\alpha} \rightarrow x_{\alpha}\left(U_{\alpha}\right)$ onto open subsets of $\mathbb{R}^{n}$ such that, whenever $U_{\alpha} \cap U_{\beta} \neq \emptyset$,

$$
x_{\alpha} \circ x_{\beta}^{-1}: x_{\beta}\left(U_{\alpha} \cap U_{\beta}\right) \rightarrow x_{\alpha}\left(U_{\alpha} \cap U_{\beta}\right)
$$

is a $C^{r}$ diffeomorphism.

Each pair $\left(U_{\alpha}, x_{\alpha}\right)$ called a chart.

Write $x_{\alpha}=\left(x^{1}, \ldots, x^{n}\right)$. The $x^{i}: U_{\alpha} \rightarrow \mathbb{R}$ are coordinates.

\subsubsection{Examples}

1. Any open subset $U \subset \mathbb{R}^{n}$ is a $C^{\infty}$ manifold with a single chart $\left(U, 1_{U}\right)$.

2. Contemplate the unit sphere $S^{n}=\left\{v \in \mathbb{R}^{n+1}:\|v\|=1\right\}$ in $\mathbb{R}^{n+1}$. Orthogonal projection provides a homeomorphism of any open hemisphere onto the open unit ball in some hyperplane $\mathbb{R}^{n} \subset \mathbb{R}^{n+1}$. The sphere is covered by the $(2 n+2)$ hemispheres lying on either side of the coordinate hyperplanes and in this way becomes a $C^{\infty}$ manifold (exercise!).

3. A good supply of manifolds is provided by the following version of the Implicit Function Theorem [6]:

Theorem. Let $f: \Omega \subset \mathbb{R}^{n} \rightarrow \mathbb{R}$ be a $C^{r}$ function $(r \geq 1)$ and $c \in \mathbb{R}$ $a$ regular value, that is, $\nabla f(x) \neq 0$, for all $x \in f^{-1}\{c\}$.

Then $f^{-1}\{c\}$ is a $C^{r}$ manifold.

Exercise. Apply this to $f(x)=\|x\|^{2}$ to get a less tedious proof that $S^{n}$ is a manifold.

\footnotetext{
${ }^{1}$ This means that there is a countable base for the topology of $M$.
} 
4. An open subset of a manifold is a manifold in its own right with charts $\left(U_{\alpha} \cap U,\left.x_{\alpha}\right|_{U_{\alpha} \cap U}\right)$.

\subsubsection{Functions and maps}

A continuous function $f: M \rightarrow \mathbb{R}$ is $C^{r}$ if each $f \circ x_{\alpha}^{-1}: x_{\alpha}\left(U_{\alpha}\right) \rightarrow \mathbb{R}$ is a $C^{r}$ function of the open set $x_{\alpha}\left(U_{\alpha}\right) \subset \mathbb{R}^{n}$.

We denote the vector space of all such functions by $C^{r}(M)$.

Example. Any coordinate function $x^{i}: U_{\alpha} \rightarrow \mathbb{R}$ is $C^{r}$ on $U_{\alpha}$.

Exercise. The restriction of any $C^{r}$ function on $\mathbb{R}^{n+1}$ to the sphere $S^{n}$ is $C^{r}$ on $S^{n}$.

In the same way, a continuous map $\phi: M \rightarrow N$ of $C^{r}$ manifolds is $C^{r}$ if, for all charts $(U, x),(V, y)$ of $M$ and $N$ respectively, $y \circ \phi \circ x^{-1}$ is $C^{r}$ on its domain of definition.

A slicker formulation? is that $h \circ \phi \in C^{r}(M)$, for all $h \in C^{r}(M)$.

At this point, having made all the definitions, we shall stop pretending to be anything other than Differential Geometers and henceforth take $r=\infty$.

\subsection{Tangent vectors and derivatives}

We now know what functions on a manifold are and it is our task to differentiate them. This requires some less than intuitive definitions so let us step back and remind ourselves of what differentiation involves.

Let $f: \Omega \subset \mathbb{R}^{n} \rightarrow \mathbb{R}$ and contemplate the derivative of $f$ at some $x \in \Omega$. This is a linear map $\mathrm{d} f_{x}: \mathbb{R}^{n} \rightarrow \mathbb{R}$. However, it is better for us to take a dual point of view and think of $\mathbf{v} \in \mathbb{R}^{n}$ is a linear map $\mathbf{v}: C^{\infty}(M) \rightarrow \mathbb{R}$ by

$$
\mathbf{v} f \stackrel{\text { def }}{=} \mathrm{d} f_{x}(\mathbf{v}) \text {. }
$$

The Leibniz rule gives us

$$
\mathbf{v}(f g)=f(x) \mathbf{v}(g)+\mathbf{v}(f) g(x) .
$$

Fact. Any linear $\mathbf{v}: C^{\infty}(\Omega) \rightarrow \mathbb{R}$ satisfying (1.1) arises this way.

Now let $M$ be a manifold. The preceding analysis may give some motivation to the following

\footnotetext{
${ }^{2}$ It requires a little machinery, in the shape of bump functions, to see that this is an equivalent formulation.
} 
Definition. A tangent vector at $m \in M$ is a linear map $\xi: C^{\infty}(M) \rightarrow \mathbb{R}$ such that

$$
\xi(f g)=f(m) \xi(g)+\xi(f) g(m)
$$

for all $f, g \in C^{\infty}(M)$.

Denote by $M_{m}$ the vector space of all tangent vectors at $m$.

Here are some examples

1. For $\gamma: I \rightarrow M$ a (smooth) path with $\gamma(t)=m$, define $\gamma^{\prime}(t) \in M_{m}$ by

$$
\gamma^{\prime}(t) f=(f \circ \gamma)^{\prime}(t)
$$

Fact. All $\xi \in M_{m}$ are of the form $\gamma^{\prime}(t)$ for some path $\gamma$.

2. Let $(U, x)$ be a chart with coordinates $x^{1}, \ldots, x^{n}$ and $x(m)=p \in \mathbb{R}^{n}$.

Define $\partial_{i \mid m} \in M_{m}$ by

$$
\partial_{i \mid m} f=\left.\frac{\partial\left(f \circ x^{-1}\right)}{\partial x^{i}}\right|_{p}
$$

Fact. $\partial_{1 \mid m}, \ldots, \partial_{n \mid m}$ is a basis for $M_{m}$.

3. For $p \in U \subset \mathbb{R}^{n}$ open, we know that $U_{p}$ is canonically isomorphic to $\mathbb{R}^{n}$ via

$$
\mathbf{v} f=\mathrm{d} f_{p}(\mathbf{v})
$$

for $\mathbf{v} \in \mathbb{R}^{n}$.

4. Let $M=f^{-1}\{c\}$ be a regular level set of $f: \Omega \subset \mathbb{R}^{n} \rightarrow \mathbb{R}$. One can show that $M_{m}$ is a linear subspace of $\Omega_{m} \cong \mathbb{R}^{n}$. Indeed, under this identification,

$$
M_{m}=\left\{\mathbf{v} \in \mathbb{R}^{n}: \mathbf{v} \perp \nabla f_{m}\right\} .
$$

Now that we have got our hands on tangent vectors, the definition of the derivative of a function as a linear map on tangent vectors is almost tautological:

Definition. For $f \in C^{\infty}(M)$, the derivative $\mathrm{d} f_{m}: M_{m} \rightarrow \mathbb{R}$ of $f$ at $m \in M$ is defined by

$$
\mathrm{d} f_{m}(\xi)=\xi f
$$


We note:

1. Each $\mathrm{d} f_{m}$ is a linear map and the Leibniz Rule holds:

$$
\mathrm{d}(f g)_{m}=g(m) \mathrm{d} f_{m}+f(m) \mathrm{d} g_{m} .
$$

2. By construction, this definition coincides with the usual one when $M$ is an open subset of $\mathbb{R}^{n}$.

Exercise. If $f$ is a constant map on a manifold $M$, show that each $\mathrm{d} f_{m}=0$.

The same circle of ideas enable us to differentiate maps between manifolds:

Definition. For $\phi: M \rightarrow N$ a smooth map of manifolds, the tangent map $\mathrm{d} \phi_{m}: M_{m} \rightarrow N_{\phi(m)}$ at $m \in M$ is the linear map defined by

$$
\mathrm{d} \phi_{m}(\xi) f=\xi(f \circ \phi),
$$

for $\xi \in M_{m}$ and $f \in C^{\infty}(N)$.

Exercise. Prove the chain rule: for $\phi: M \rightarrow N$ and $\psi: N \rightarrow Z$ and $m \in M$,

$$
\mathrm{d}(\psi \circ \phi)_{m}=\mathrm{d} \psi_{\phi(m)} \circ \mathrm{d} \phi_{m} .
$$

Exercise. View $\mathbb{R}$ as a manifold (with a single chart!) and let $f: M \rightarrow \mathbb{R}$. We now have two competing definitions of $\mathrm{d} f_{m}$. Show that they coincide.

The tangent bundle of $M$ is the disjoint union of the tangent spaces:

$$
T M=\coprod_{m \in M} M_{m} .
$$

\subsection{Vector fields}

Definition. A vector field is a linear map $X: C^{\infty}(M) \rightarrow C^{\infty}(M)$ such that

$$
X(f g)=f(X g)+g(X f) .
$$

Let $\Gamma(T M)$ denote the vector space of all vector fields on $M$.

We can view a vector field as a map $X: M \rightarrow T M$ with $X(m) \in M_{m}$ : indeed, we have

$$
X_{\mid m} \in M_{p}
$$


where

$$
X_{\mid m} f=(X f)(m) .
$$

In fact, vector fields can be shown to be exactly those maps $X: M \rightarrow T M$ with $X(m) \in M_{m}$ which satisfy the additional smoothness constraint that for each $f \in C^{\infty}(M)$, the function $m \mapsto X(m) f$ is also $C^{\infty}$.

The Lie bracket of $X, Y \in \Gamma(T M)$ is $[X, Y]: C^{\infty}(M) \rightarrow C^{\infty}(M)$ given by

$$
[X, Y] f=X(Y f)-Y(X f) .
$$

The point of this definition is contained in the following

Exercise. Show that $[X, Y] \in \Gamma(T M)$ also.

The Lie bracket is interesting for several reasons. Firstly it equips $\Gamma(T M)$ with the structure of a Lie algebra; secondly, it, and operators derived from it, are the only differential operators that can be defined on an arbitrary manifold without imposing additional structures such as special coordinates, a Riemannian metric, a complex structure or a symplectic form.

There is an extension of the notion of vector field that we shall need later on:

Definition. Let $\phi: M \rightarrow N$ be a map. A vector field along $\phi$ is a map $X: M \rightarrow T N$ with

$$
X(m) \in N_{\phi(m)},
$$

for all $m \in M$, which additionally satisfies a smoothness assumption that we shall gloss over.

Denote by $\Gamma\left(\phi^{-1} T N\right)$ the vector space of all vector fields along $\phi$.

Here are some examples:

1. If $c: I \rightarrow N$ is a smooth path then $c^{\prime} \in \Gamma\left(\phi^{-1} T N\right)$.

2. More generally, for $\phi: M \rightarrow N$ and $X \in \Gamma(T M), \mathrm{d} \phi(X) \in \Gamma\left(\phi^{-1} T N\right)$. Here, of course,

$$
\mathrm{d} \phi(X)(m)=\mathrm{d} \phi_{m}\left(X_{\mid m}\right)
$$

3. For $Y \in \Gamma(T N), Y \circ \phi \in \Gamma\left(\phi^{-1} T N\right)$. 


\subsection{Connections}

We would like to differentiate vector fields but as they take values in different vector spaces at different points, it is not so clear how to make difference quotients and so derivatives. What is needed is some extra structure: a connection which should be thought of as a "directional derivative" for vector fields.

Definition. A connection on $T M$ is a bilinear map

$$
\begin{aligned}
T M \times \Gamma(T M) & \rightarrow T M \\
(\xi, X) & \mapsto \nabla_{\xi} X
\end{aligned}
$$

such that, for $\xi \in M_{m}, X, Y \in \Gamma(T M)$ and $f \in C^{\infty}(M)$,

1. $\nabla_{\xi} X \in M_{m}$

2. $\nabla_{\xi}(f X)=(\xi f) X_{\mid m}+f(m) \nabla_{\xi} X$

3. $\nabla_{X} Y \in \Gamma(T M)$.

A connection on $T M$ comes with some additional baggage in the shape of two multilinear maps:

$$
\begin{aligned}
& T_{m}: M_{m} \times M_{m} \rightarrow M_{m} \\
& R_{m}: M_{m} \times M_{m} \times M_{m} \rightarrow M_{m}
\end{aligned}
$$

given by

$$
\begin{aligned}
T_{m}(\xi, \eta) & =\nabla_{\xi} Y-\nabla_{\eta} X-[X, Y]_{\mid m} \\
R_{m}(\xi, \eta) \zeta & =\nabla_{\eta} \nabla_{X} Z-\nabla_{\xi} \nabla_{Y} Z-\nabla_{[Y, X]_{\mid m}}
\end{aligned}
$$

where $X, Y, Z \in \Gamma(T M)$ with $X_{\mid m}=\xi, Y_{\mid m}=\eta$ and $Z_{\mid m}=\zeta$.

$T_{m}$ and $R_{m}$ are, respectively, the torsion and curvature at $m$ of $\nabla$.

Fact. $R$ and $T$ are well-defined - they do not depend of the choice of vector fields $X, Y$ and $Z$ extending $\xi, \eta$ and $\zeta$.

We have some trivial identities:

$$
\begin{aligned}
T(\xi, \eta) & =-T(\eta, \xi) \\
R(\xi, \eta) \zeta & =-R(\eta, \xi) \zeta .
\end{aligned}
$$

and, if each $T_{m}=0$, we have the less trivial First Bianchi Identity:

$$
R(\xi, \eta) \zeta+R(\zeta, \xi) \eta+R(\eta, \zeta) \xi=0 .
$$


A connection $\nabla$ on $T N$ induces a similar operator on vector fields along a map $\phi: M \rightarrow N$. To be precise, there is a unique bilinear map

$$
\begin{aligned}
T M \times \Gamma\left(\phi^{-1} T N\right) & \rightarrow T N \\
(\xi, X) & \mapsto \phi^{-1} \nabla_{\xi} X
\end{aligned}
$$

such that, for $\xi \in M_{m}, X \in \Gamma(T M), Y \in \Gamma\left(\phi^{-1} T N\right)$ and $f \in C^{\infty}(M)$,

1. $\phi^{-1} \nabla_{\xi} Y \in N_{\phi(m)}$;

2. $\phi^{-1} \nabla_{\xi}(f Y)=(\xi f) Y_{\mid \phi(m)}+f(m) \phi^{-1} \nabla_{\xi} Y$;

3. $\phi^{-1} \nabla_{X} Y \in \Gamma\left(\phi^{-1} T N\right)$ (this is a smoothness assertion);

4. If $Z \in \Gamma(T N)$ then $Z \circ \phi \in \Gamma\left(\phi^{-1} T N\right)$ and

$$
\phi^{-1} \nabla_{\xi}(Z \circ \phi)=\nabla_{\mathrm{d} \phi_{m}(\xi)} Z \text {. }
$$

$\phi^{-1} \nabla$ is the pull-back of $\nabla$ by $\phi$. The first three properties just say that $\phi^{-1} \nabla$ behaves like $\nabla$, it is the last that essentially defines it in a unique way.

\section{Analysis on Riemannian manifolds}

\section{$2.1 \quad$ Riemannian manifolds}

A rich and useful geometry arises if we equip each $M_{m}$ with an inner product:

Definition. A Riemannian metric $g$ on $M$ is an inner product $g_{m}$ on each $M_{m}$ such that, for all vector fields $X$ and $Y$, the function

$$
m \mapsto g_{m}\left(X_{\mid m}, Y_{\mid m}\right)
$$

is smooth.

A Riemannian manifold is a pair $(M, g)$ with $M$ a manifold and $g$ a metric on $M$.

Here are some (canonical) examples:

1. Let $\left(\right.$, ) denote the inner product on $\mathbb{R}^{n}$.

An open $U \subset \mathbb{R}^{n}$ gets a Riemannian metric via $U_{m} \cong \mathbb{R}^{n}$ :

$$
g_{m}(v, w)=(v, w)
$$


2. Let $S^{n} \subset \mathbb{R}^{n+1}$ be the unit sphere. Then $S_{m}^{n} \cong m^{\perp} \subset \mathbb{R}^{n+1}$ and so gets a metric from the inner product on $\mathbb{R}^{n+1}$.

3. Let $D^{n} \subset \mathbb{R}^{n}$ be the open unit disc but define a metric by

$$
g_{z}(v, w)=\frac{4(v, w)}{\left(1-|z|^{2}\right)^{2}}
$$

$\left(D^{n}, g\right)$ is hyperbolic space.

Much of the power of Riemannian geometry comes from the fact that there is a canonical choice of connection. Consider the following two desirable properties for a connection $\nabla$ on $(M, g)$ :

1. $\nabla$ is metric: $X g(Y, Z)=g\left(\nabla_{X} Y, Z\right)+g\left(Y, \nabla_{X} Z\right)$.

2. $\nabla$ is torsion-free: $\nabla_{X} Y-\nabla_{Y} X=[X, Y]$

Theorem. There is a unique torsion-free metric connection on any Riemannian manifold.

Proof. Assume that $g$ is metric and torsion-free. Then

$$
\begin{aligned}
g\left(\nabla_{X} Y, Z\right) & =X g(Y, Z)-g\left(Y, \nabla_{X} Z\right) \\
& =X g(Y, Z)-g(Y,[X, Z])-g\left(Y, \nabla_{Z} X\right) \ldots
\end{aligned}
$$

and eventually we get

$$
\begin{aligned}
2 g\left(\nabla_{X} Y, Z\right)=X g(Y, Z) & +Y g(Z, Y)-Z g(X, Y) \\
& -g(X,[Y, Z])+g(Y,[Z, X])+g(Z,[X, Y]) .
\end{aligned}
$$

This formula shows uniqueness and, moreover, defines the desired connection.

This connection is the Levi-Civita connection of $(M, g)$.

For detailed computations, it is sometimes necessary to express the metric and Levi-Civita connection in terms of local coordinates. So let $(U, x)$ be a chart and $\partial_{1}, \ldots, \partial_{n}$ be the corresponding vector fields on $U$. We now define $g_{i j} \in C^{\infty}(U)$ by

$$
g_{i j}=g\left(\partial_{i}, \partial_{j}\right)
$$

and Christoffel symbols $\Gamma_{i j}^{k} \in C^{\infty}(U)$ by

$$
\nabla_{\partial_{i}} \partial_{j}=\sum_{k} \Gamma_{i j}^{k} \partial_{k}
$$


(Recall that $\partial_{1 \mid m}, \ldots, \partial_{n \mid m}$ form a basis for $M_{m}$.)

Now let $\left(g^{i j}\right)$ be the matrix inverse to $\left(g_{i j}\right)$. Then the formula (2.1) for $\nabla$ reads:

$$
\Gamma_{i j}^{k}=\frac{1}{2} \sum_{l} g^{k l}\left(\partial_{i} g_{j l}+\partial_{j} g_{l i}-\partial_{l} g_{i j}\right)
$$

since the bracket terms $\left[\partial_{i}, \partial_{j}\right]$ vanish (exercise!).

\subsection{Differential operators}

The metric and Levi-Civita connection of a Riemannian manifold are precisely the ingredients one needs to generalise the familiar operators of vector calculus:

The gradient of $f \in C^{\infty}(M)$ is the vector field $\operatorname{grad} f$ such that, for $Y \in$ $\Gamma(T M)$,

$$
g(\operatorname{grad} f, Y)=Y f .
$$

Similarly, the divergence of $X \in \Gamma(T M)$ is the function $\operatorname{div} f \in C^{\infty}(M)$ defined by:

$$
(\operatorname{div} f)(m)=\operatorname{trace}\left(\xi \rightarrow \nabla_{\xi} X\right)
$$

Finally, we put these together to introduce the hero of this volume: the Laplacian of $f \in C^{\infty}(M)$ is the function

$$
\Delta f=\operatorname{div} \operatorname{grad} f .
$$

In a chart $(U, x)$, set $\mathbf{g}=\operatorname{det}\left(g_{i j}\right)$. Then

$$
\operatorname{grad} f=\sum_{i, j} g^{i j}\left(\partial_{i} f\right) \partial_{j}
$$

and, for $X=\sum_{i} X_{i} \partial_{i}$,

$$
\begin{aligned}
\operatorname{div} X & =\sum_{i}\left(\partial_{i} X_{i}+\sum_{j} \Gamma_{i j}^{i} X_{j}\right) \\
& =\frac{1}{\sqrt{\mathbf{g}}} \sum_{j} \partial_{j}\left(\sqrt{\mathbf{g}} X_{j}\right) .
\end{aligned}
$$

Here we have used $\sum_{i} \Gamma_{i j}^{i}=\left(\partial_{j} \sqrt{\mathbf{g}}\right) / \sqrt{\mathbf{g}}$ which the Reader is invited to deduce from (2.2) together with the well-known formula for a matrix-valued function $A$ :

$$
\mathrm{d} \ln \operatorname{det} A=\operatorname{trace} A^{-1} \mathrm{~d} A \text {. }
$$


In particular, we conclude that

$$
\Delta f=\frac{1}{\sqrt{\mathbf{g}}} \sum_{i, j} \partial_{i}\left(\sqrt{\mathbf{g}} g^{i j} \partial_{j} f\right)=\sum_{i, j} g^{i j}\left(\partial_{i} \partial_{j} f-\Gamma_{i j}^{k} \partial_{k} f\right) .
$$

\subsection{Integration on Riemannian manifolds}

\subsubsection{Riemannian measure}

$(M, g)$ has a canonical measure $d V$ on its Borel sets which we define in steps: First let $(U, x)$ be a chart and $f: U \rightarrow \mathbb{R}$ a measureable function. We set

$$
\int_{U} f d V=\int_{x(U)}\left(f \circ x^{-1}\right) \sqrt{\mathbf{g} \circ x^{-1}} \mathrm{~d} x^{1} \ldots \mathrm{d} x^{n} .
$$

Fact. The change of variables formula ensures that this integral is welldefined on the intersection of any two charts.

To get a globally defined measure, we patch things together with a partition of unity: since $M$ is second countable and locally compact, it follows that every open cover of $M$ has a locally finite refinement. A partition of unity for a locally finite open cover $\left\{U_{\alpha}\right\}$ is a family of functions $\phi_{\alpha} \in C^{\infty}(M)$ such that

1. $\operatorname{supp}\left(\phi_{\alpha}\right) \subset U_{\alpha}$

2. $\sum_{\alpha} \phi_{\alpha}=1$.

Theorem. [6, Theorem 1.11] Any locally finite cover has a partition of unity.

Armed with this, we choose a locally finite cover of $M$ by charts $\left\{\left(U_{\alpha}, x_{\alpha}\right)\right\}$, a partition of unity $\left\{\phi_{\alpha}\right\}$ for $\left\{U_{\alpha}\right\}$ and, for measurable $f: M \rightarrow \mathbb{R}$, set

$$
\int_{M} f d V=\sum_{\alpha} \int_{U_{\alpha}} \phi_{\alpha} f d V .
$$

Fact. This definition is independent of all choices. 


\subsubsection{The Divergence Theorem}

Let $X \in \Gamma(T M)$ have support in a chart $(U, x)$.

$$
\begin{aligned}
\int_{M} \operatorname{div} X d V & =\int_{U} \frac{1}{\sqrt{\mathbf{g}}} \partial_{i}\left(\sqrt{\mathbf{g}} X_{i}\right) d V \\
& =\int_{x(U)}\left(\partial_{i} \sqrt{\mathbf{g}} X_{i}\right) \circ x^{-1} \mathrm{~d} x^{1} \ldots \mathrm{d} x^{n} \\
& =\int_{x(U)} \frac{\partial}{\partial x^{i}}\left(\sqrt{\mathbf{g}} X_{i}\right) \circ x^{-1} \mathrm{~d} x^{1} \ldots \mathrm{d} x^{n}=0 .
\end{aligned}
$$

A partition of unity argument immediately gives:

Divergence Theorem I. Any compactly supported vector field $X$ on $M$ has

$$
\int_{M} \operatorname{div} X d V=0
$$

Just as in vector calculus, the divergence theorem quickly leads to Green's formulae. Indeed, for $f, h \in C^{\infty}(M), X \in \Gamma(T M)$ one easily verifies:

$$
\operatorname{div}(f X)=f \operatorname{div} X+g(\operatorname{grad} f, X)
$$

whence

$$
\begin{aligned}
\operatorname{div}(f \operatorname{grad} h) & =f \Delta h+g(\operatorname{grad} h, \operatorname{grad} f) \\
\Delta(f h) & =f \Delta h+2 g(\operatorname{grad} h, \operatorname{grad} f)+h \Delta f .
\end{aligned}
$$

The divergence theorem now gives us Green's Formulae:

Theorem. For $f, h \in C^{\infty}(M)$ with at least one of $f$ and $h$ compactly supported:

$$
\begin{aligned}
& \int_{M} h \Delta f d V=-\int_{M} g(\operatorname{grad} f, \operatorname{grad} h) d V \\
& \int_{M} h \Delta f d V=\int_{M} f \Delta h d V .
\end{aligned}
$$

\subsubsection{Boundary terms}

Supposed that $M$ is oriented and that $\Omega \subset M$ is an open subset with smooth boundary $\partial \Omega$. Thus $\partial \Omega$ is a smooth manifold with

1. a Riemannian metric inherited via $(\partial \Omega)_{m} \subset M_{m}$; 
2. a Riemannian measure $d A$;

3. a unique outward-pointing normal unit vector field $\nu$.

With these ingredients, one has:

Divergence Theorem II. Any compactly supported $X$ on $M$ has

$$
\int_{\Omega} \operatorname{div} X d V=\int_{\partial \Omega} g(X, \nu) d A
$$

and so Green's Formulae:

Theorem. For $f, h \in C^{\infty}(M)$ with at least one of $f$ and $h$ compactly supported:

$$
\begin{aligned}
\int_{\Omega} h \Delta f+\langle\operatorname{grad} f, \operatorname{grad} h\rangle d V & =\int_{\partial \Omega} h\langle\nu, \operatorname{grad} f\rangle d A \\
\int_{\Omega} h \Delta f-\int_{\Omega} f \Delta h d V & =\int_{\partial \Omega} h\langle\nu, \operatorname{grad} f\rangle d A-\int_{\partial \Omega} f\langle\nu, \operatorname{grad} h\rangle d A
\end{aligned}
$$

where we have written $\langle$,$\rangle for g($,$) .$

In particular

$$
\int_{\Omega} \Delta f d V=\int_{\partial \Omega} \nu f d V
$$

\section{Geodesics and curvature}

In the classical geometry of Euclid, a starring role is played by the straight lines. Viewed as paths of shortest length between two points, these may be generalised to give a distinguished family of paths, the geodesics, on any Riemannian manifold. Geodesics provide a powerful tool to probe the geometry of Riemannian manifolds.

Notation. Let $(M, g)$ be a Riemannian manifold. For $\xi, \eta \in M_{m}$, write

$$
g(\xi, \eta)=\langle\xi, \eta\rangle, \quad \sqrt{g(\xi, \xi)}=|\xi| .
$$

\section{$3.1(M, g)$ is a metric space}

A piece-wise $C^{1}$ path $\gamma:[a, b] \rightarrow M$ has length $L(\gamma)$ :

$$
L(\gamma)=\int_{a}^{b}\left|\gamma^{\prime}(t)\right| \mathrm{d} t
$$


Exercise. The length of a path is invariant under reparametrisation.

Recall that $M$ is connected and so[ path-connected. For $p, q \in M$, set

$$
d(p, q)=\inf \{L(\gamma): \gamma:[a, b] \rightarrow M \text { is a path with } \gamma(a)=p, \gamma(b)=q\} .
$$

One can prove:

- $(M, d)$ is a metric space.

- The metric space topology coincides with the original topology on $M$.

The key points here are the definiteness of $d$ and the assertion about the topologies. For this, it is enough to work in a precompact open subset of a chart $U$ where one can prove the existence of $K_{1}, K_{2} \in \mathbb{R}$ such that

$$
K_{1} \sum_{1 \leq i \leq n} \xi_{i}^{2} \leq \sum_{i, j} g_{i j} \xi_{i} \xi_{j} \leq K_{2} \sum_{1 \leq i \leq n} \xi_{i}^{2} .
$$

From this, one readily sees that, on such a subset, $d$ is equivalent to the Euclidean metric on $U$.

\subsection{Parallel vector fields and geodesics}

Let $c: I \rightarrow M$ be a path. Recall the pull-back connection $c^{-1} \nabla$ on the space $\Gamma\left(c^{-1} T M\right)$ of vector fields along $c$. This connection gives rise to a differential operator

$$
\nabla_{t}: \Gamma\left(c^{-1} T M\right) \rightarrow \Gamma\left(c^{-1} T M\right)
$$

by

$$
\nabla_{t} Y=\left(c^{-1} \nabla\right)_{\partial_{1}} Y
$$

where $\partial_{1}$ is the coordinate vector field on $I$.

Note that since $\nabla$ is metric, we have

$$
\langle X, Y\rangle^{\prime}=\left\langle\nabla_{t} X, Y\right\rangle+\left\langle X, \nabla_{t} Y\right\rangle
$$

for $X, Y \in \Gamma\left(c^{-1} T M\right)$.

Definition. $X \in \Gamma\left(c^{-1} T M\right)$ is parallel if $\nabla_{t} X=0$.

The existence and uniqueness results for linear ODE give:

\footnotetext{
${ }^{3}$ Manifolds are locally path-connected!
} 
Proposition. For $c:[a, b] \rightarrow M$ and $U_{0} \in M_{c(a)}$, there is unique parallel vector field $U$ along $c$ with

$$
U(a)=U_{0}
$$

If $Y_{1}, Y_{2}$ are parallel vector fields along $c$, then all $\left\langle Y_{i}, Y_{j}\right\rangle$ and, in particular, $\left|Y_{i}\right|$ are constant.

Definition. $\gamma: I \rightarrow M$ is a geodesic if $\gamma^{\prime}$ is parallel:

$$
\nabla_{t} \gamma^{\prime}=0
$$

It is easy to prove that, for a geodesic $\gamma$ :

- $\left|\gamma^{\prime}\right|$ is constant.

- If $\gamma$ is a geodesic, so is $t \mapsto \gamma(s t)$ for $s \in \mathbb{R}$.

The existence and uniqueness results for ODE give:

1. For $\xi \in M_{m}$, there is a maximal open interval $I_{\xi} \subset \mathbb{R}$ on which there is a unique geodesic $\gamma_{\xi}: I_{\xi} \rightarrow M$ such that

$$
\begin{aligned}
\gamma_{\xi}(0) & =m \\
\gamma_{\xi}^{\prime}(0) & =\xi
\end{aligned}
$$

2. $(t, \xi) \mapsto \gamma_{\xi}(t)$ is a smooth map $I_{\xi} \times M_{m} \rightarrow M$.

3. $\gamma_{s \xi}(t)=\gamma_{\xi}(s t)$.

Let us collect some examples:

1. $M=\mathbb{R}^{n}$ with its canonical metric. The geodesic equation reduces to:

$$
\frac{\mathrm{d}^{2} \gamma}{\mathrm{d} t^{2}}=0
$$

and we conclude that geodesics are straight lines.

2. $M=S^{n}$ and $\xi$ is a unit vector in $M_{m}=m^{\perp}$. Contemplate reflection in the 2-plane spanned by $m$ and $\xi$ : this induces a map $\Phi: S^{n} \rightarrow S^{n}$ which preserves the metric and so $\nabla$ also while it fixes $m$ and $\xi$. Thus, if $\gamma$ is a geodesic so is $\Phi \circ \gamma$ and the uniqueness part of the ODE yoga forces $\Phi \circ \gamma_{\xi}=\gamma_{\xi}$. Otherwise said, $\gamma_{\xi}$ lies in the plane spanned by $m$ and $\xi$ and so lies on a great circle. 
To get further, recall that $\left|\gamma_{\xi}^{\prime}\right|=|\xi|=1$ which implies:

$$
\gamma_{\xi}(t)=(\cos t) m+(\sin t) \xi .
$$

A similar argument shows that the unique parallel vector field $U$ along $\gamma_{\xi}$ with $U(0)=\eta \perp \xi$ is given by

$$
U \equiv \eta
$$

3. $M=D^{n}$ with the hyperbolic metric and $\xi$ is a unit vector in $M_{0} \cong \mathbb{R}^{n}$. Again, symmetry considerations force $\gamma_{\xi}$ to lie on the straight line through 0 in the direction of $\xi$ and then $\left|\gamma_{\xi}^{\prime}\right|=1$ gives:

$$
\gamma_{\xi}(t)=(2 \tanh t / 2) \xi .
$$

Similarly, the parallel vector field along $\gamma_{\xi}$ with $U(0)=\eta \perp \xi$ is given by

$$
U(t)=\frac{1}{\cosh ^{2} t / 2} \eta
$$

\subsection{The exponential map}

\subsubsection{Normal coordinates}

Set $\mathcal{U}_{m}=\left\{\xi \in M_{m}: 1 \in I_{\xi}\right\}$ and note that $\mathcal{U}_{m}$ is a star-shaped open neighbourhood of $0 \in M_{m}$. We define the exponential map $\exp _{m}: \mathcal{U}_{m} \rightarrow M$ by

$$
\exp _{m}(\xi)=\gamma_{\xi}(1)
$$

Observe that, for all $t \in I_{\xi}$,

$$
\exp _{m}(t \xi)=\gamma_{t \xi}(1)=\gamma_{\xi}(t)
$$

and differentiating this with respect to $t$ at $t=0$ gives

$$
\xi=\gamma_{\xi}^{\prime}(0)=\left(\mathrm{d} \exp _{m}\right)_{0}(\xi)
$$

so that $\left(\operatorname{dexp}_{m}\right)_{0}=1_{M_{m}}$. Thus, by the inverse function theorem, $\exp _{m}$ is a local diffeomorphism whose inverse is a chart.

Indeed, if $e_{1}, \ldots, e_{n}$ is an orthonormal basis of $M_{m}$, we have normal coordinates $x^{1}, \ldots, x^{n}$ given by

$$
x^{i}=\left\langle\left(\exp _{m}\right)^{-1}, e_{i}\right\rangle
$$

for which

$$
\begin{aligned}
g_{i j}(m) & =\delta_{i j} \\
\Gamma_{i j}^{k}(m) & =0 .
\end{aligned}
$$




\subsubsection{The Gauss Lemma}

Let $\xi, \eta \in M_{m}$ with $|\xi|=1$ and $\xi \perp \eta$.

The Gauss Lemma says:

$$
\left\langle\left(d \exp _{m}\right)_{t \xi} \eta, \gamma_{\xi}^{\prime}(t)\right\rangle=0 .
$$

Thus $\gamma_{\xi}$ intersects the image under $\exp _{m}$ of spheres in $M_{m}$ orthogonally.

As an application, let us show that geodesics are locally length-minimising. For this, choose $\delta>0$ sufficiently small that

$$
\exp _{m}: B(0, \delta) \subset M_{m} \rightarrow M
$$

is a diffeomorphism onto an open set $U \subset M$. Let $c: I \rightarrow U$ be a path from $m$ to $p \in U$ and let $\gamma: I \rightarrow U$ be the geodesic from $m$ to $p$ : thus $\gamma$ is the image under $\exp _{m}$ of a radial line segment in $B(0, \delta)$.

Write

$$
c(t)=\exp _{m}(r(t) \xi(t))
$$

with $r: I \rightarrow \mathbb{R}$ and $\xi: I \rightarrow S^{n} \subset M_{m}$. Now

$$
\begin{aligned}
\left\langle c^{\prime}(t), c^{\prime}(t)\right\rangle & =\left(r^{\prime}\right)^{2}+r^{2}\left\langle\left(\mathrm{~d}_{\exp }\right)_{r \xi} \xi^{\prime},\left(\mathrm{d}_{\exp _{m}}\right)_{r \xi} \xi^{\prime}\right\rangle+2 r r^{\prime}\left\langle\left(\mathrm{d} \exp _{m}\right)_{r \xi} \xi^{\prime}, \gamma_{\xi}^{\prime}\right\rangle \\
& =\left(r^{\prime}\right)^{2}+r^{2}\left\langle\left(\mathrm{~d}_{\exp _{m}}\right)_{r \xi} \xi^{\prime},\left(\mathrm{d}_{\exp _{m}}\right)_{r \xi} \xi^{\prime}\right\rangle
\end{aligned}
$$

by the Gauss lemma (since $\xi^{\prime} \perp \xi$ ). In particular,

$$
\left\langle c^{\prime}(t), c^{\prime}(t)\right\rangle \geq\left(r^{\prime}\right)^{2} .
$$

Taking square roots and integrating gives:

$$
L(c) \geq \int_{a}^{b}\left|r^{\prime}\right| \mathrm{d} t \geq\left|\int_{a}^{b} r^{\prime} \mathrm{d} t\right|=|r(b)-r(a)|=L(\gamma) .
$$

From this we conclude:

$$
L(\gamma)=d(m, p)
$$

and

$$
B_{d}(m, \delta)=\exp _{m} B(0, \delta) .
$$

Definition. A geodesic $\gamma$ is minimising on $[a, b] \subset I_{\gamma}$ if

$$
L\left(\gamma_{\mid[a, b]}\right)=d(\gamma(a), \gamma(b)) .
$$

We have just seen that any geodesic is minimising on sufficiently small intervals. 


\subsubsection{The Hopf-Rinow Theorem}

Definition. $(M, g)$ is geodesically complete if $I_{\xi}=\mathbb{R}$, for any $\xi \in \mathbb{R}$.

This only depends on the metric space structure of $(M, d)$ :

Theorem (Hopf-Rinow). The following are equivalent:

1. $(M, g)$ is geodesically complete.

2. For some $m \in M$, $\exp _{m}$ is a globally defined surjection $M_{m} \rightarrow M$.

3. Closed, bounded subsets of $(M, d)$ are compact.

4. $(M, d)$ is a complete metric space.

In this situation, one can show that any two points of $M$ can be joined by a minimising geodesic.

\subsection{Sectional curvature}

Let $\sigma \subset M_{m}$ be a 2-plane with orthonormal basis $\xi, \eta$.

The sectional curvature $\mathcal{K}(\sigma)$ of $\sigma$ is given by

$$
\mathcal{K}(\sigma)=\langle R(\xi, \eta) \xi, \eta\rangle
$$

Facts:

- This definition is independent of the choice of basis of $\sigma$.

- $\mathcal{K}$ determines the curvature tensor $R$.

Definition. $(M, g)$ has constant curvature $\kappa$ if $\mathcal{K}(\sigma)=\kappa$ for all 2-planes $\sigma$ in $T M$.

In this case, we have

$$
R(\xi, \eta) \zeta=\kappa\{\langle\xi, \zeta\rangle \eta-\langle\eta, \zeta\rangle \xi\}
$$

$\mathcal{K}$ is a function on the set (in fact manifold) $G_{2}(T M)$ of all 2-planes in all tangent spaces $M_{m}$ of $M$. A diffeomorphism $\Phi: M \rightarrow M$ induces $\mathrm{d} \Phi: T M \rightarrow T M$ which is a linear isomorphism on each tangent space and so gives a mapping $\hat{\Phi}: G_{2}(T M) \rightarrow G_{2}(T M)$. Suppose now that $\Phi$ is an isometry:

$$
\left\langle\mathrm{d} \Phi_{m}(\xi), \mathrm{d} \Phi_{m}(\eta)\right\rangle=\langle\xi, \eta\rangle,
$$


for all $\xi, \eta \in M_{m}, m \in M$. Since an isometry preserves the metric, it will preserve anything built out of the metric such as the Levi-Civita connection and its curvature. In particular, we have

$$
\mathcal{K} \circ \hat{\Phi}=\mathcal{K} .
$$

It is not too difficult to show that, for our canonical examples, the group of all isometries acts transitively on $G_{2}(T M)$ so that $\mathcal{K}$ is constant. Thus we arrive at the following examples of manifolds of constant curvature:

1. $\mathbb{R}^{n}$.

2. $S^{n}(r)$.

3. $D^{n}(\rho)$ with metric

$$
g_{i j}=\frac{4 \delta_{i j}}{\left(1-|z|^{2} / \rho^{2}\right)^{2}}
$$

It can be shown that these exhaust all complete, simply-connected possibilities.

\subsection{Jacobi fields}

Definition. Let $\gamma: I \rightarrow M$ be a unit speed geodesic. Say $Y \in \Gamma\left(\gamma^{-1} T M\right)$ is a Jacobi field along $\gamma$ if

$$
\nabla_{t}^{2} Y+R\left(\gamma^{\prime}, Y\right) \gamma^{\prime}=0
$$

Once again we wheel out the existence and uniqueness theorems for ODE which tell us:

Proposition. For $Y_{0}, Y_{1} \in M_{\gamma(0)}$, there is a unique Jacobi field $Y$ with

$$
\begin{aligned}
Y(0) & =Y_{0} \\
\left(\nabla_{t} Y\right)(0) & =Y_{1}
\end{aligned}
$$

Jacobi fields are infinitesimal variations of $\gamma$ through a family of geodesics. Indeed, suppose that $h: I \times(-\epsilon, \epsilon) \rightarrow M$ is a variation of geodesics: that is, each $\gamma_{s}: t \rightarrow h(t, s)$ is a geodesic. Set $\gamma=\gamma_{0}$ and let

$$
Y=\left.\frac{\partial h}{\partial s}\right|_{s=0} \in \Gamma\left(\gamma^{-1} T M\right) .
$$


Let $\partial_{t}$ and $\partial_{s}$ denote the coordinate vector fields on $I \times(-\epsilon, \epsilon)$ and set $D=h^{-1} \nabla$. Since each $\gamma_{s}$ is a geodesic, we have

$$
D_{\partial_{t}} \frac{\partial h}{\partial t}=0
$$

whence

$$
D_{\partial_{s}} D_{\partial_{t}} \frac{\partial h}{\partial t}=0
$$

The definition of the curvature tensor, along with the fact that $\left[\partial_{s}, \partial_{t}\right]=0$, allows us to write

$$
0=D_{\partial_{s}} D_{\partial_{t}} \frac{\partial h}{\partial t}=D_{\partial_{t}} D_{\partial_{s}} \frac{\partial h}{\partial t}+R\left(\frac{\partial h}{\partial t}, \frac{\partial h}{\partial s}\right) \frac{\partial h}{\partial t} .
$$

Moreover, it follows from the fact that $\nabla$ is torsion-free that

$$
D_{\partial_{s}} \frac{\partial h}{\partial t}=D_{\partial_{t}} \frac{\partial h}{\partial s}
$$

so that

$$
0=\left(D_{\partial_{t}}\right)^{2} \frac{\partial h}{\partial s}+R\left(\frac{\partial h}{\partial t}, \frac{\partial h}{\partial s}\right) \frac{\partial h}{\partial t} .
$$

Setting $s=0$, this last becomes

$$
\left(\nabla_{t}\right)^{2} Y+R\left(\gamma^{\prime}, Y\right) \gamma^{\prime}=0 .
$$

Fact. All Jacobi fields arise this way.

Let us contemplate an example which will compute for us the (constant) value of $\mathcal{K}$ for hyperbolic space: let $\left(D^{n}, g\right)$ be hyperbolic space and consider a path $\xi:(-\epsilon, \epsilon) \rightarrow S^{n-1} \subset D_{0}$ with $\xi^{\prime}(0)=\eta \perp \xi(0)$.

We set $h(t, s)=\gamma_{\xi(s)}(t)=(2 \tanh t / 2) \xi(s)$-a variation of geodesics through 0 . We then have a Jacobi field $Y$ along $\gamma=\gamma_{\xi(0)}$ :

$$
\begin{aligned}
Y(t)=\left.\frac{\partial h}{\partial s}\right|_{s=0} & =2(\tanh t / 2) \eta \\
& =\sinh t\left(\eta / \cosh ^{2} t / 2\right) \\
& =\sinh t U(t)
\end{aligned}
$$

where $U$ is a unit length parallel vector field along $\gamma$.

We therefore have:

$$
\left(\nabla_{t}\right)^{2} Y=\sinh ^{\prime \prime} t U(t)=\sinh t U(t)
$$


whence

$$
U+R\left(\gamma^{\prime}, U\right) \gamma^{\prime}=0 .
$$

Take an inner product with $U$ to get

$$
\mathcal{K}\left(\gamma^{\prime} \wedge U\right)=-1
$$

and so conclude that $\left(D^{n}, g\right)$ has constant curvature -1 .

The same argument (that is, differentiate the image under $\exp _{m}$ of a family of straight lines through the origin) computes Jacobi fields in normal coordinates:

Theorem. For $\xi \in M_{m}$, the Jacobi field $Y$ along $\gamma_{\xi}$ with

$$
\begin{aligned}
Y(0) & =0 \\
\left(\nabla_{t} Y\right)(0) & =\eta \in M_{m}
\end{aligned}
$$

is given by

$$
Y(t)=(\mathrm{d} \exp )_{t \xi} t \eta .
$$

\subsection{Conjugate points and the Cartan-Hadamard theorem}

Let $\xi \in M_{p}$ and let $\gamma=\gamma_{\xi}: I_{\xi} \rightarrow \mathbb{R}$. We say that $q=\gamma\left(t_{1}\right)$ is conjugate to $p$ along $\gamma$ if there is a non-zero Jacobi field $Y$ with

$$
Y(0)=Y\left(t_{1}\right)=0 .
$$

In view of the theorem just stated, this happens exactly when $\left(\mathrm{dexp}_{p}\right)_{t_{1} \xi}$ is singular.

Theorem (Cartan-Hadamard). If $(M, g)$ is complete and $\mathcal{K} \leq 0$ then no $p \in M$ has conjugate points.

Proof. Suppose that $Y$ is a Jacobi field along some geodesic $\gamma$ with $Y(0)=$ $Y\left(t_{1}\right)=0$. Then

$$
\begin{aligned}
0 & =\int_{0}^{t_{1}}\left\langle\nabla_{t}^{2} Y+R\left(\gamma^{\prime}, Y\right) \gamma^{\prime}, Y\right\rangle \mathrm{d} t \\
& =-\int_{0}^{t_{1}}\left|\nabla_{t} T\right|^{2} \mathrm{~d} t+\int_{0}^{t_{1}} \mathcal{K}\left(\gamma^{\prime} \wedge Y\right)|Y|^{2} \mathrm{~d} t
\end{aligned}
$$

where we have integrated by parts and used $Y(0)=Y\left(t_{1}\right)=0$ to kill the boundary term. Now both summands in this last equation are non-negative and so must vanish. In particular,

$$
\nabla_{t} Y=0
$$

so that $Y$ is parallel whence $|Y|$ is constant giving eventually $Y \equiv 0$. 
From this we see that, under the hypotheses of the theorem, each $\exp _{m}$ is a local diffeomorphism and, with a little more work, one can show that $\exp _{m}: M_{m} \rightarrow M$ is a covering map. Thus:

Corollary. If $(M, g)$ is complete and $\mathcal{K} \leq 0$ then

1. if $\pi_{1}(M)=1$ then $M$ is diffeomorphic to $\mathbb{R}^{n}$.

2. In any case, the universal cover of $M$ is diffeomorphic to $\mathbb{R}^{n}$ whence $\pi_{k}(M)=1$ for all $k \geq 2$.

Analysis of this kind is the starting point of one of the central themes of modern Riemannian geometry: the interplay between curvature and topology.

\section{The Bishop volume comparison theorem}

Our aim is to prove a Real Live Theorem in Riemannian geometry: the theorem is of considerable interest in its own right and proving it will exercise everything we have studied in these notes.

We begin by collecting some ingredients.

\subsection{Ingredients}

\subsubsection{Ricci curvature}

Definition. The Ricci tensor at $m \in M$ is the bilinear map Ric : $M_{m} \times$ $M_{m} \rightarrow \mathbb{R}$ given by

$$
\begin{aligned}
\operatorname{Ric}(\xi, \eta) & =\operatorname{trace}(\zeta \mapsto R(\xi, \zeta) \eta) \\
& =\sum_{i}\left\langle R\left(\xi, e_{i}\right) \eta, e_{i}\right\rangle
\end{aligned}
$$

where $e_{1}, \ldots, e_{n}$ is an orthonormal basis of $M_{m}$.

Exercise. The Ricci tensor is symmetric: $\operatorname{Ric}(\xi, \eta)=\operatorname{Ric}(\eta, \xi)$.

Example. If $(M, g)$ has dimension $n$ and constant curvature $\kappa$ then

$$
\operatorname{Ric}=(n-1) \kappa g .
$$

The Ricci tensor, being only bilinear, is much easier to think about than the curvature tensor. On the other hand, being only an average of sectional curvatures, conditions of the Ricci tensor say much less about the topology of the underlying manifold. For example, here is an amazing theorem of Lohkamp [4]: 
Theorem. Any manifold of dimension at least 3 admits a complete metric with $\mathrm{Ric}<0$ (that is Ric is neagtive definite).

\subsubsection{Cut locus}

Henceforth, we will take $M$ to be complete of dimension $n$.

For $\xi \in M_{m}$ with $|\xi|=1$, define $c(\xi) \in \mathbb{R}^{+} \cup\{\infty\}$ by

$$
\begin{aligned}
c(\xi) & =\sup \left\{t:\left.\gamma_{\xi}\right|_{[0, t]} \text { is minimising }\right\} \\
& =\sup \left\{t: d\left(m, \gamma_{\xi}(t)\right)=t\right\} .
\end{aligned}
$$

The cut locus $C_{m}$ of $m$ is given by

$$
C_{m}=\exp _{m}\left\{c(\xi) \xi: \xi \in S^{n-1} \subset M_{m}, c(\xi)<\infty\right\}
$$

while $\mathcal{D}_{m}=\left\{t \xi: \xi \in S^{n-1} \subset M_{m}, t \in[0, c(\xi))\right\}$ and

$$
D_{m}=\exp _{m} \mathcal{D}_{m} \text {. }
$$

We have:

- $M=D_{m} \cup C_{m}$ is a disjoint union.

- $\exp _{m}: \mathcal{D}_{m} \rightarrow D_{m}$ is a diffeomorphism.

- $\int_{C_{m}} d V=0$.

These facts have practical consequences for integration on $M:$ for $f: M \rightarrow \mathbb{R}$ integrable,

$$
\begin{aligned}
\int_{M} f d V & =\int_{\mathcal{D}_{m}} f(\exp (x)) \sqrt{\mathbf{g}} \mathrm{d} x^{1} \ldots \mathrm{d} x^{n} \\
& =\int_{S^{n-1}} \int_{0}^{c(\xi)} f(\exp (r \xi)) \mathbf{a}(r, \xi) \mathrm{d} r \mathrm{~d} \xi
\end{aligned}
$$

where $x^{1}, \ldots, x^{n}$ are orthonormal coordinates on $\mathcal{D}_{m}$ and $\mathrm{d} \xi$ is Lebesgue measure on $S^{n-1} \subset M_{m}$.

Example. For $\kappa \in \mathbb{R}$, let $S_{\kappa}: \mathbb{R} \rightarrow \mathbb{R}$ solve

$$
\begin{gathered}
S_{\kappa}^{\prime \prime}+\kappa S_{\kappa}=0 \\
S_{\kappa}(0)=0, \quad S_{\kappa}^{\prime}(0)=1
\end{gathered}
$$

Then, if $(M, g)$ has constant curvature $\kappa$,

$$
\mathbf{a}(r, \xi)=S_{\kappa}^{n-1}(r) .
$$




\subsection{Bishop's Theorem}

\subsubsection{Manifesto}

Fix $\kappa \in \mathbb{R}$ and $m \in M_{m}$.

Let $V(m, r)$ denote the volume of $B_{d}(m, r) \subset M$ and $V_{\kappa}(r)$ the volume of a radius $r$ ball in a complete simply-connected $n$-dimensional space of constant curvature $\kappa$.

Suppose that $\operatorname{Ric}(\xi, \xi) \geq(n-1) \kappa g(\xi, \xi)$ for all $\xi \in T M$. For each $\xi \in M_{m}$ of unit length, define $a_{\xi}:(0, c(\xi)) \rightarrow \mathbb{R}$ by

$$
a_{\xi}(t)=\mathbf{a}(t, \xi) .
$$

We will prove that

$$
\frac{a_{\xi}^{\prime}}{a_{\xi}} \leq(n-1) \frac{S_{\kappa}^{\prime}}{S_{\kappa}}
$$

As a consequence, we will see that

$$
V(m, r) \leq V_{\kappa}(r)
$$

and even that $V(m, r) / V_{\kappa}(r)$ is decreasing with respect to $r$.

\subsubsection{Laplacian of the distance function}

Our strategy will be to identify the radial logarithmic derivative of a with the Laplacian of the distance from $m$. We will then be able to apply a formula of Lichnerowicz to derive a differential inequality for $a_{\xi}$.

So view $r$ as a function on $M$ :

$$
r(x)=d(m, x)
$$

Then

Proposition. $\mathbf{a}^{-1} \partial \mathbf{a} / \partial r=\Delta r \circ \exp _{m}$.

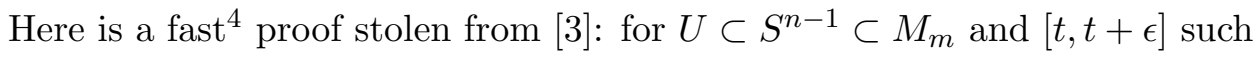
that

$$
\Omega_{t, \epsilon}=\left\{\exp _{m}(r \xi): r \in[t, t+\epsilon], \xi \in U\right\} \subset D_{m}
$$

\footnotetext{
${ }^{4}$ Isaac Chavel rightly objects that this proof is all a bit too slick. See his contribution to this volume for a more down to earth proof.
} 
we have:

$$
\int_{\Omega_{t, \epsilon}} \Delta r d V=\int_{[t, t+\epsilon] \times U}\left(\Delta \circ \exp _{m}\right) \mathbf{a} \mathrm{d} r \mathrm{~d} \xi
$$

However, the divergence theorem gives

$$
\begin{aligned}
\int_{\Omega_{t, \epsilon}} \Delta d V=\int_{\partial \Omega_{t, \epsilon}}\langle\operatorname{grad} r, \nu\rangle d A & =\int_{U} \mathbf{a}(t+\epsilon) \mathrm{d} \xi-\int_{U} \mathbf{a}(t) \mathrm{d} \xi \\
& =\int_{U} \int_{t}^{t+\epsilon} \frac{\partial \mathbf{a}}{\partial r}(r, \xi) \mathrm{d} r \mathrm{~d} \xi
\end{aligned}
$$

Here we have used that $\langle\operatorname{grad} r, \nu\rangle=\nu r=1$ along the spherical parts of $\partial \Omega_{t, \epsilon}$ and vanishes along the radial parts.

Thus

$$
\int_{[t, t+\epsilon] \times U}\left(\Delta \circ \exp _{m}\right) \mathbf{a} \mathrm{d} r \mathrm{~d} \xi=\int_{[t, t+\epsilon] \times U} \frac{\partial \mathbf{a}}{\partial r}(r, \xi) \mathrm{d} r \mathrm{~d} \xi
$$

and, since $t, \epsilon$ and $U$ were arbitrary, we get

$$
\mathbf{a}\left(\Delta \circ \exp _{m}\right)=\frac{\partial \mathbf{a}}{\partial r}
$$

as required.

\subsubsection{Lichnerowicz' formula}

For $X \in \Gamma(T M)$, define $|\nabla X|^{2}$ by

$$
|\nabla X|^{2}(m)=\sum_{i}\left|\nabla_{e_{i}} X\right|^{2}
$$

where $e_{1}, \ldots, e_{n}$ is an orthonormal basis of $M_{m}$-this is independent of choices.

We now have

Lichnernowicz' Formula. Let $f: M \rightarrow \mathbb{R}$ then

$$
\frac{1}{2} \Delta|\operatorname{grad} f|^{2}=|\nabla \operatorname{grad} f|^{2}+\langle\operatorname{grad} \Delta f, \operatorname{grad} f\rangle+\operatorname{Ric}(\operatorname{grad} f, \operatorname{grad} f) .
$$

The proof of this is an exercise (really!) but here are some hints to get you started: the basic identity

$$
X Y f-Y X f=[X, Y]
$$


along with the fact that $\nabla$ is metric and torsion-free gives:

$$
\left\langle\nabla_{X} \operatorname{grad} f, Y\right\rangle=\left\langle\nabla_{Y} \operatorname{grad} f, X\right\rangle
$$

from which you can deduce that

$$
\frac{1}{2} \operatorname{grad}|\operatorname{grad} f|^{2}=\nabla_{\operatorname{grad} f} \operatorname{grad} f
$$

whence

$$
\begin{aligned}
\frac{1}{2} \Delta|\operatorname{grad} f|^{2} & =\operatorname{div} \nabla_{\operatorname{grad} f} \operatorname{grad} f \\
& =\sum_{i}\left\langle\nabla_{e_{i}} \nabla_{\operatorname{grad} f} \operatorname{grad} f, e_{i}\right\rangle .
\end{aligned}
$$

Now make repeated use of the metric property of $\nabla$ and use the definition of $R$ to change the order of the differentiations ...

As an application, put $f=r$. Thanks to the Gauss lemma, grad $f=\partial_{r}$ so that $|\operatorname{grad} f|=1$ and the Lichnerowicz formula reads:

$$
0=|\nabla \operatorname{grad} r|^{2}+\partial_{r} \Delta r+\operatorname{Ric}\left(\partial_{r}, \partial_{r}\right) .
$$

On the image of $\gamma_{\xi}$, we have

$$
\partial_{r} \Delta r=\left(a_{\xi}^{\prime} / a_{\xi}\right)^{\prime}=a_{\xi}^{\prime \prime} / a_{\xi}-(\Delta r)^{2}
$$

and plugging this into (4.1) gives

$$
0=a_{\xi}^{\prime \prime} / a_{\xi}-(\Delta r)^{2}+|\nabla \operatorname{grad} r|^{2}+\operatorname{Ric}\left(\partial_{r}, \partial_{r}\right)
$$

or, defining $b$ by $b^{n-1}=a_{\xi}$ so that $(n-1) b^{\prime} / b=a_{\xi}^{\prime} / a_{\xi}$,

$$
(n-1) b^{\prime \prime} / b+\operatorname{Ric}\left(\partial_{r}, \partial_{r}\right)=-\left(|\nabla \operatorname{grad} r|^{2}-\frac{1}{n-1}(\Delta r)^{2}\right) .
$$

\subsubsection{Estimates and comparisons}

We now show that the right hand side of (4.2) has a sign: choose an orthonormal basis $e_{1}, \ldots, e_{n}$ of $M_{\gamma_{\xi}(t)}$ with $e_{1}=\partial_{r}$. Then

$$
\begin{aligned}
\Delta r & =\sum\left\langle\nabla_{e_{i}} \operatorname{grad} r, e_{i}\right\rangle \\
& =\sum_{i \geq 2}\left\langle\nabla_{e_{i}} \operatorname{grad} r, e_{i}\right\rangle
\end{aligned}
$$

since $\nabla_{\partial_{r}} \operatorname{grad} r=\nabla_{t} \gamma_{\xi}^{\prime}=0$. 
Two applications of the Cauchy-Schwarz inequality give

$$
(\Delta r)^{2} \leq\left(\sum_{i \geq 2}\left|\nabla_{e_{i}} \operatorname{grad} r\right|\right)^{2} \leq(n-1) \sum_{i \geq 2}\left|\nabla_{e_{i}} \operatorname{grad} r\right|^{2}
$$

so that

$$
|\nabla \operatorname{grad} r|^{2}-\frac{1}{n-1}(\Delta r)^{2} \geq 0
$$

Thus (4.2) gives

$$
(n-1) b^{\prime \prime} / b+\operatorname{Ric}\left(\partial_{r}, \partial_{r}\right) \leq 0
$$

and, under the hypotheses of Bishop's theorem, we have

$$
b^{\prime \prime} / b \leq-\kappa .
$$

We now make a simple comparison argument: $b>0$ on $(0, c(\xi))$ so we have

$$
\begin{gathered}
b^{\prime \prime}+\kappa b \leq 0 \\
b(0)=0, \quad b^{\prime}(0)=1 .
\end{gathered}
$$

On the other hand, set $\bar{b}=S_{\kappa}$ so that

$$
\begin{gathered}
\bar{b}^{\prime \prime}+\kappa \bar{b}=0 \\
\bar{b}(0)=0, \quad \bar{b}^{\prime}(0)=1
\end{gathered}
$$

We now see that, so long as $\bar{b} \geq 0$, we have

$$
\bar{b} b^{\prime \prime}-\bar{b}^{\prime \prime} b \leq 0
$$

or, equivalently,

$$
\left(b^{\prime} \bar{b}-\bar{b}^{\prime} b\right)^{\prime} \leq 0 .
$$

In view of the initial conditions, we conclude:

$$
b^{\prime} \bar{b}-\bar{b}^{\prime} b \leq 0 \text {. }
$$

Let us pause to observe that at the first zero of $\bar{b}$ (if there is one), $\bar{b}^{\prime}<0$ so that, by (4.3),$b \leq 0$ also. Since $b>0$ on $(0, c(\xi))$, we deduce that $\bar{b}>0$ there also包.

We therefore conclude from (4.3) that on $(0, c(\xi))$ we have

$$
b^{\prime} / b \leq \bar{b}^{\prime} / \bar{b},
$$

or, equivalently,

$$
a_{\xi}^{\prime} / a_{\xi} \leq(n-1) S_{\kappa}^{\prime} / S_{\kappa} .
$$

\footnotetext{
${ }^{5}$ For $\kappa>0$, this reasoning puts an upper bound on the length of $(0, c(\xi))$ and thus, eventually, on the diameter of $M$. This leads to a proof of the Bonnet-Myers theorem.
} 


\subsubsection{Baking the cake}

Equation (4.4) reads

$$
\ln \left(a_{\xi} / S_{\kappa}^{n-1}\right)^{\prime} \leq 0
$$

so that, $a_{\xi} / S_{\kappa}^{n-1}$ is decreasing and, in view of the initial conditions,

$$
a_{\xi} \leq S_{\kappa}^{n-1} .
$$

Thus:

$$
\begin{aligned}
V(m, r) & =\int_{S^{n-1}} \int_{0}^{\min (c(\xi), r)} a_{\xi} \mathrm{d} r \mathrm{~d} \xi \\
& \leq \int_{S^{n-1}} \int_{0}^{\min (c(\xi), r)} S_{\kappa}^{n-1} \mathrm{~d} r \mathrm{~d} \xi=V_{\kappa}(r) .
\end{aligned}
$$

This is Bishop's theorem.

Our final statement is due to Gromov [Z] and is a consequence of a simple lemma:

Lemma ([2]). If $f, g>0$ with $f / g$ decreasing then

$$
\int_{0}^{r} f / \int_{0}^{r} g
$$

is decreasing also.

With this in hand, we see that, for $r_{1}<r_{2}$,

$$
\int_{0}^{r_{1}} a_{\xi} \mathrm{d} r / \int_{0}^{r_{1}} S_{\kappa}^{n-1} \mathrm{~d} r \leq \int_{0}^{r_{2}} a_{\xi} \mathrm{d} r / \int_{0}^{r_{2}} S_{\kappa}^{n-1} \mathrm{~d} r .
$$

Integrating this over $S^{n-1}$, noting that the denominators are independent of $\xi$, gives finally that $V(m, r) / V_{\kappa}(r)$ is decreasing.

\section{References}

[1] I. Chavel, Riemannian geometry - a modern introduction, Cambridge University Press, Cambridge, 1993. [1

[2] J. Cheeger, M. Gromov, and M. Taylor, Finite propagation speed, kernel estimates for functions of the Laplace operator, and the geometry of complete Riemannian manifolds, J. Differential Geom. 17 (1982), no. 1, 15-53. 28, 28 
[3] S. Gallot, D. Hulin, and J. Lafontaine, Riemannian geometry, SpringerVerlag, Berlin, 1987. 1, 1, 24

[4] J. Lohkamp, Metrics of negative Ricci curvature, Ann. of Math. (2) 140 (1994), no. 3, 655-683. 22

[5] M. Spivak, Calculus on manifolds. A modern approach to classical theorems of advanced calculus, W. A. Benjamin, Inc., New York-Amsterdam, 1965. प

[6] F.W. Warner, Foundations of differentiable manifolds and Lie groups, Scott, Foresman and Co., Glenview, Ill.-London, 1971. [1, 2, 11] 